\title{
EVOLUTIONARY GAME THEORETIC RESOURCE ALLOCATION SIMULATION FOR MOLECULAR COMMUNICATION
}

\author{
Caglar Koca ${ }^{1}$, Meltem Civas $^{2}$, Ozgur B. Akan ${ }^{1,2}$ \\ ${ }^{1}$ Internet of Everthing (IoE) Group, Department of Engineering, University of Cambridge, UK, ${ }^{2}$ Next-generation and \\ Wireless Communications Laboratory (NWCL), Department of Electrical and Electronics Engineering, Koç University, \\ Istanbul, Turkey
}

NOTE: Corresponding author: Caglar Koca, ck542@cam.ac.uk

\begin{abstract}
Molecular Communication (MC) is an emerging technology using molecules to transfer information between nanomachines. In this paper, we approach the resource allocation problem in Molecular Nano-networks (MCN) from the perspective of evolutionary game theory. In particular, we consider an MCN as an organism having three types of nodes acting as a sensor, relay, and sink, respectively. The resources are distributed among the nodes according to an evolutionary process, which relies on the selection of the most successful organisms followed by creating their offspring iteratively. In this regard, the success of an organism is measured by the total number of dropped messages during its life cycle. To illustrate the evolution procedure, we design a toy problem, and then solve it analytically and using the evolution approach for comparison. We further simulate the performance of the evolution approach on randomly generated organisms. The results reveal the potential of evolutionary game theory tools to improve the transmission performance of MCNs.
\end{abstract}

Keywords - Evolutionary game theory, molecular communication, nano-networks, resource allocation

\section{INTRODUCTION}

The Internet of Bio-Nano Things (IoBNT) is a novel paradigm based on the interconnection of nanoscale devices and biological entities. It enables novel applications ranging from intra-body sensing and actuator networks for the treatment and diagnosis of various diseases to the monitoring and control of environmental pollution $[1,2]$. Molecular Communication (MC), which is inspired by the natural communication between biological entities, emerges as a promising communication technology to realize nano-networks for IoBNT applications [3], and recently recognized as an effective abstraction tool for understanding several diseases $[4,5,6]$.

Research efforts in MC mainly focused on physical channel modeling, modulation, coding, and detection techniques $[7,8,9,10,11]$. Developing MC Nano-networks (MCN) acting and communicating cooperatively to perform IoBNT tasks still poses many challenges.

One of the most prominent challenges in MC is resource allocation. Relying on molecular dispersion instead of electromagnetic waves, MC devices need to preserve both energy and molecules to continue their operation. As a result, resource allocation is a more prominent problem for MC compared to EM, where preserving and/or harvesting energy is enough to guarantee high transmission efficiency.

Realizing the importance of resource allocation in MC, several works are proposed to overcome this problem. In [12], the optimal number of molecules released by the transmitter and the optimal detection threshold of the receiver minimizing the error probability of each hop in a multi-hop MCN are derived. In [13], the joint optimization of molecular resource allocation and relay location is investigated to improve the error performance of a cooperative MC system. Similarly, in [14], the optimal molecule allocation among molecular receivers is determined for a cooperative MC system. In [15], information molecules with different diffusion coefficients are considered optimizing molecular resource allocation in molecular multiple access networks.

MC is the primary communication mode for all organisms. It covers both short ranges as in synaptic communication and long ranges as in pheromones. It is used for both fast acting purposes such as adrenaline and slow acting purposes such as growth. DNA itself is an application of MC that transfers information through ages. Therefore, we turn to evolution, the driving factor of the MC utilization diversity, to find answers relating to the resource allocation problem. One way to tap into the power of evolution is using evolutionary game theory.

Game theory has been used as a tool to model networking problems and to investigate communication efficiency of MCN. Game theory principles can help one explore communication among molecular nanomachines when it is hard to obtain analytical solutions because of the size of MCN. Jiang et al [16] propose a game-theoretic approach for distributed research allocation for MC networks based on Nash equilibrium and Nash bargaining schemes. In [17], evolutionary game theory tools are used to explore the effect of transmitter behaviors, namely cooperation 
or confrontation, regarding communication efficiency in MCN. Game theory has also been used to describe the behavioral dynamics of natural MCNs for sharing common resources such as bacteria populations [18] and plant microbiomes [19].

To the best of our knowledge, evolutionary game theory itself has not been considered in the literature for the MCN resource allocation problem. The success of evolutionary game theory in application to biological problems, including resource allocation in organisms [20] shows its applicability to the nature inspired MCN.

In this paper, we apply evolutionary game theory to the resource allocation problem in MCNs. The evolution procedure relies on selecting successful MCNs, where the success criterion is the total number of successful transmissions, followed by creating their offspring iteratively. By this approach, we have a population of MCNs which is generally better in terms of transmission count as we increase the iteration number. In a way, this approach resembles machine learning. In other words, our simulation behaves as an evolutionary machine learning algorithm based on mechanisms of evolutionary game theory. To illustrate the evolutionary approach, we use a toy problem for resource management in an MCN and then provide respective analytical and evolutionary solutions comparatively. We also demonstrate our simulation using a randomly generated MCN.

The rest of the paper is organized as follows. In Section 2 , we describe the system model, i.e., the operation of the MCN and evolution procedure. In Section 3, we present an analytical and an evolutionary solution for a toy problem comparatively to illustrate how evolution works. In Section 4, we simulate a randomly generated MCN, using the evolutionary approach, and then discuss its performance. Finally, we conclude the paper in Section 5.

\section{SYSTEM MODEL}

We use an organism as an MCN. The organism consists of nodes communicating with each other using MC. In Section 2.1 we describe the organism in detail and in Section 2.2 we will present the system model for MC. Finally, in Section 2.3, we will illustrate the evolution procedure.

\subsection{Organism}

An organism has three types of nodes: sensor nodes collecting information about their surroundings, $\operatorname{sink}(\mathrm{s})$ operating as gateways to the Internet, and relay nodes transmitting the information they received from the sensor nodes to the sink(s). Because of the extremely small sizes of the relay nodes, they are randomly distributed in a volume. However, sensor nodes and the sink are larger, so they are not necessarily distributed randomly. Fig. 1 depicts the organism. We made the following assumptions regarding the operation of the nodes:
- The sensor nodes have an infinite number of molecules, while the relay nodes have a finite size molecule reservoir.

- The nodes have a good knowledge of each other and the location of the sinks, i.e., nodes use a localization algorithm prior to their operation.

- The existing nodes do not cause any obstruction for the nodes behind them.

The general operation of the organism is depicted in Algorithm 1. Firstly, one of the sensor nodes emits a message (lines 10-12). When a relay node receives the message, it relays the message to another node until the message reaches the sink (line 26). If several relay nodes receive the message, a random one among them, which is closer to the sink than the previously emitting relay node (lines 14-17), transmits the message (lines 18-19). The organism is considered dead if $X$ messages are dropped consecutively or if reservoirs of $P \%$ of the relay nodes are exhausted (line 9). The latter condition is included to support the former. Without this condition, if the consecutive drop cycle is broken by a lucky transmission, at least $X$ more attempts are made, which increases the drop counts and overall performance parameters are disrupted.

\subsection{Molecular communication model}

In this work, we use a simplistic model capturing all the essential mechanisms of MC without the computational burden. As with any MC model, our model comprises information carriers, medium, transmission, and reception, as described in detail as follows.

\subsubsection{Information carrier}

As the name suggests, the information carrier is a molecule that can diffuse and propagate in a medium. The molecules do not interact with each other, and they have a constant, isotropic diffusion constant, $D$. The half-life of the molecules is assumed long compared to the propagation time and short compared to the time between consecutive transmissions of the nodes. Therefore, the molecule count does not drop during propagation to other nodes. Moreover, we assume that the channel is cleared between consecutive transmissions.

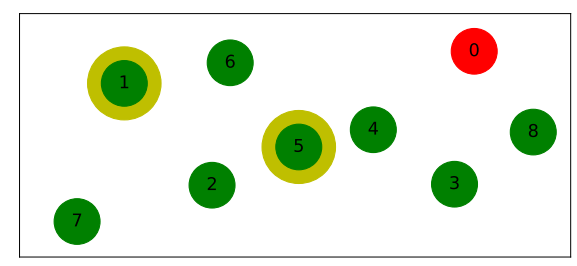

Fig. 1 - An example of an organism, with sink shown as red and sensors nodes shown with yellow rings around them. The other nodes are relay nodes. 


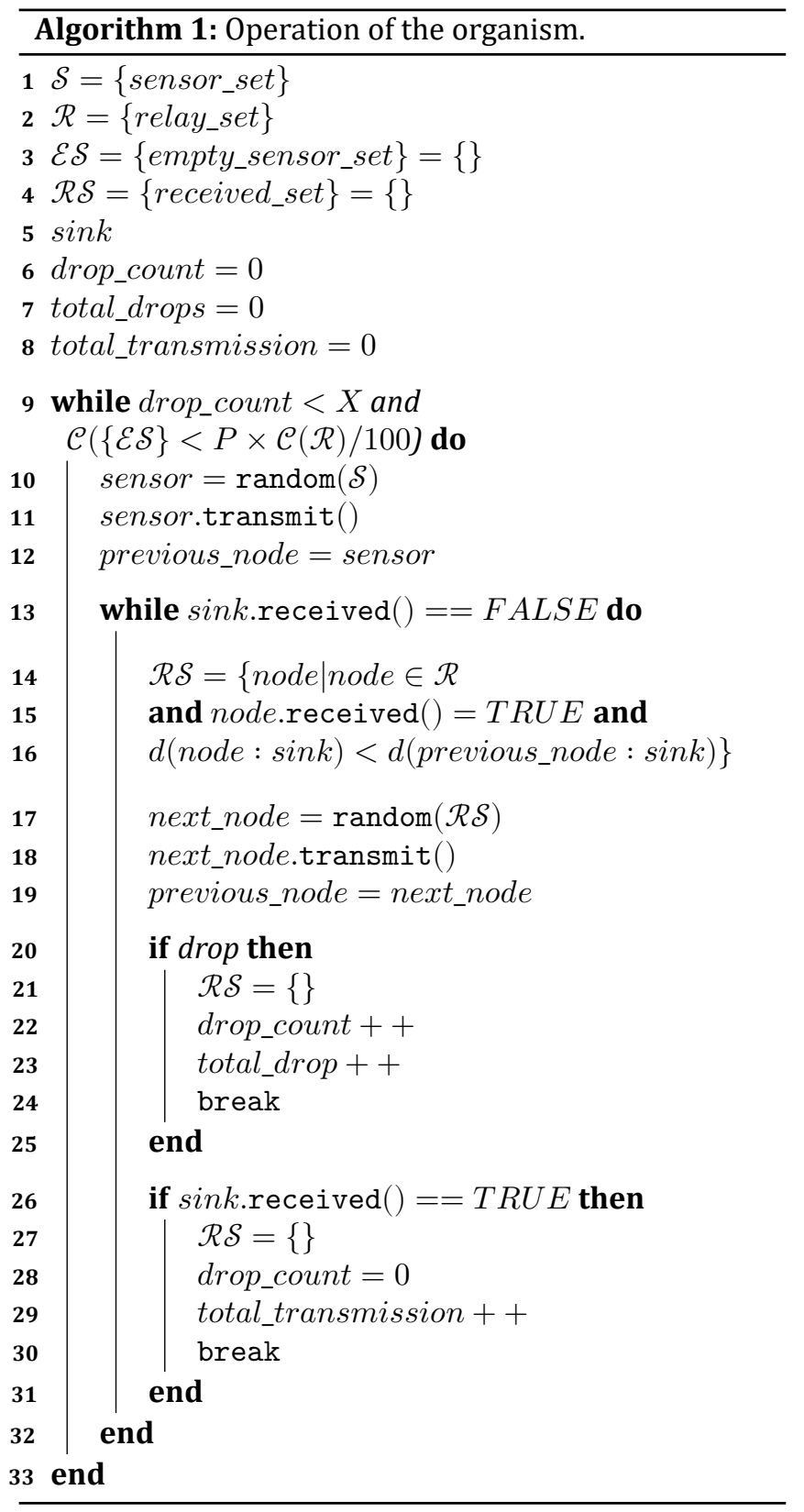

\subsubsection{Medium}

The transmission medium is a $3 \mathrm{D}$ medium where molecules can diffuse. The medium is isotropic and homogeneous, i.e., the diffusivity of the molecules does not depend on the position or direction.

\subsubsection{Transmission}

Each node in the organism is capable of receiving and transmitting messages. The nodes transmit the message by releasing vesicles containing a fixed number of molecules. The release is assumed to happen in the center of the node in an omnidirectional manner.

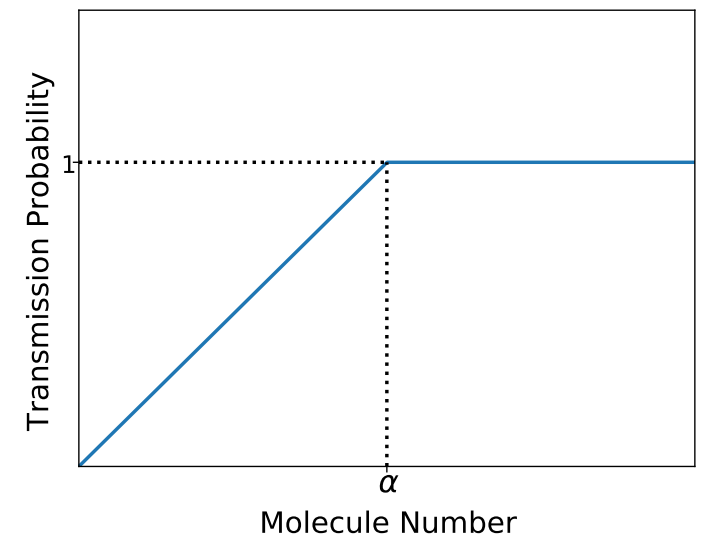

Fig. 2 - Reception behavior depending on molecule count in the vicinity.

\subsubsection{Reception}

Reception occurs when molecules are incident on the receptors located on the nodes. Considering a binding mechanism following the standard lock-and-key model, both the molecular concentration in the vicinity of the receptor and the orientation of molecules according to the receptor are important. In other words, while a single molecule with the correct orientation can bind the receptor, many molecules without the correct orientation may fail to trigger a reception. Hence, to capture the stochastic properties of reception, we use the following simple approach. If the maximum number of molecules exceeds a certain threshold, $\alpha$, the binding probability approaches 1 , so we assume successful transmission. If the number of molecules is below $\alpha$, there is a non-zero chance of successful binding. This chance is directly proportional to the molecule count in the vicinity. Fig. 2 depicts the reception behavior. We can summarize reception with (1),

$$
P_{1}(t)= \begin{cases}1, & \max _{t \rightarrow t+\Delta T} M \geq \alpha \\ 0, & \max _{t \rightarrow t+\Delta T} M=0 \\ \frac{\max _{t \rightarrow t+\Delta T} M}{\alpha}, & 0 \leq \max _{t \rightarrow t+\Delta T} M \leq \alpha\end{cases}
$$

where $\max _{t \rightarrow t+\Delta T} M$ is the maximum number of molecules in the vicinity of the node and $P_{1}(t)$ is probabilities of successfully receiving the emitted signal.

\subsection{Evolution}

As stated in Section 2.1, the organism dies after it consecutively drops messages from its sensors. The total number of dropped messages during its life cycle measures the organism's success.

The more successful organisms, which are chosen according to the selection process, then produce their offspring, i.e., the creation. Iterated selection and creation stages constitute evolution. The evolution procedure is summarized in Algorithm 2. First, as the generation dies, the 


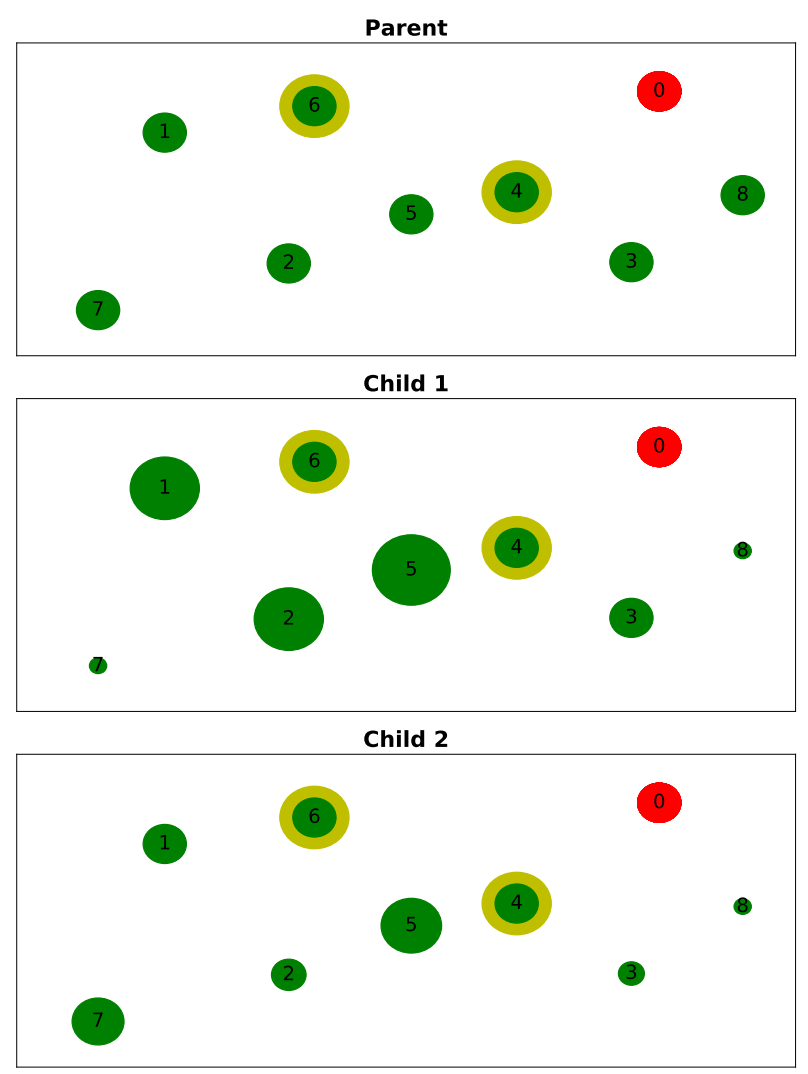

Fig. 3 - The parent, which has the same reservoir size in each node, evolves into two children. The radii of the nodes are proportional to the reservoir size. Note that for illustration purposes, the carried changes were not infinitesimal.

most successful $R$ of organisms in the generation of $N$ organisms are selected (lines 4-5). Then, the selections form their offspring (line 6). The nodes of these offspring have very similar reservoir distribution among their relay nodes compared to their parent. The resources are distributed among the relay nodes only with infinitesimal changes compared to the resource distribution of their parent. Hence, suboptimal distributions are eliminated, and better nodes are created. A parent with two offspring is presented in Fig. 3.

Note that during procreation, the resources available to each node of an organism changes by a random number $k$ such that

$$
k=\left\{-k_{\max },-k_{\max }+1 \ldots,-1,0,1, \ldots, k_{\max }\right\}
$$

If $k<0$ and the node reservoir becomes smaller than 0 , the resources are set to 0 .

Note that the total resources distributed to all nodes are kept constant. Otherwise, organisms with a higher total number of resources would dominate the others, and we could not obtain any information regarding optimum resource distribution.

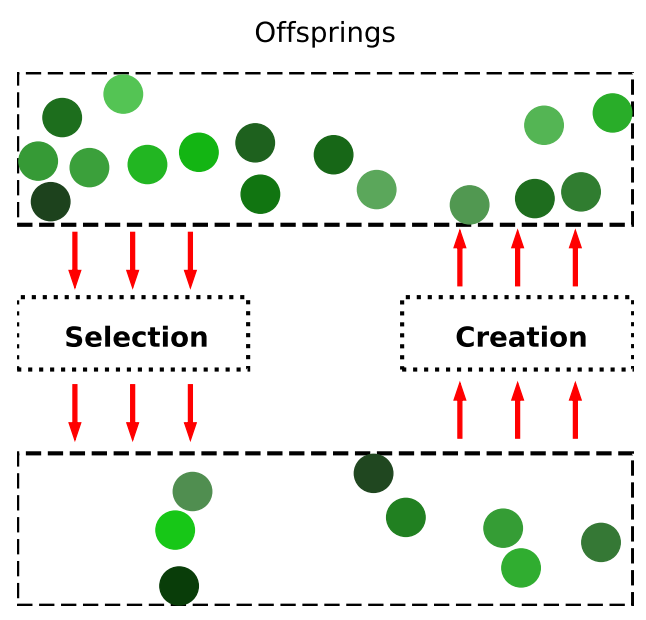

Parents

Fig. 4 - In the first stage, offspring of the previous generation are selected through the selection rules. In the second stage, selected parents create the next generation. In our approach, the selection rule is the higher total transmission count.

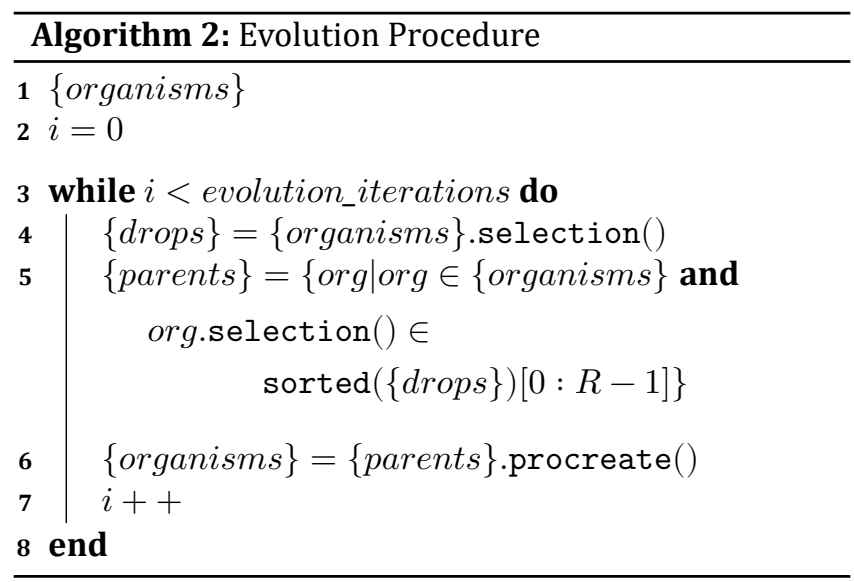

\section{EVOLUTIONARY GAME THEORY}

Evolutionary game theory is an application of game theory for evolution and population dynamics. It suggests that the collective behavior of the individuals, whether they are rational, or not plays a vital role in the survival and continuation of the species.

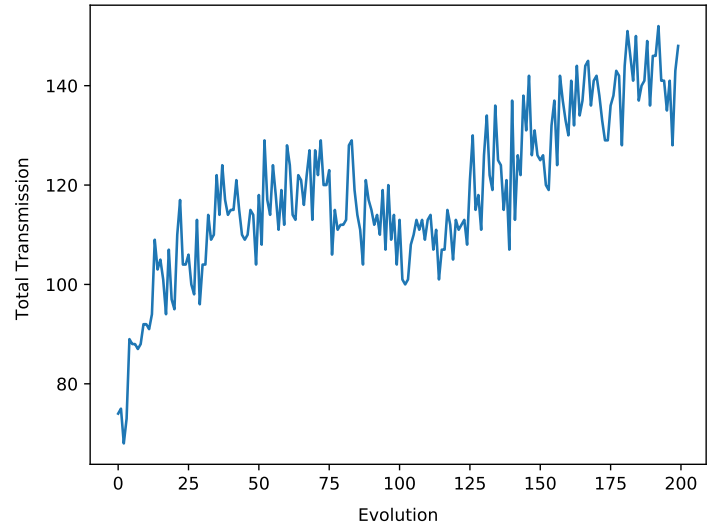

Fig. 5 - Total number of transmissions by the organism through stages of evolution. 
Evolutionary game theory is a two-stage process. In the first stage, the population members undergo a selection process depending on the individual strategies of the pop- ulation. In the second stage, selected individuals create new offspring. The phases of evolutionary game theory are depicted in Fig. 4.

In this section, we first describe the toy problem and then present an analytic solution using the simple geometry of the toy problem. Then, we demonstrate an evolutionary solution for the same problem and compare the results with the analytic solution.

\subsection{Problem description}

To illustrate how evolution works, consider the toy problem given in Fig. 6. The parent consists of one sink node, two sensor nodes, $\mathcal{S}=\left\{n_{4}, n_{6}\right\}$ and six relay nodes. The goal, as described in Algorithm 1, is either to minimize
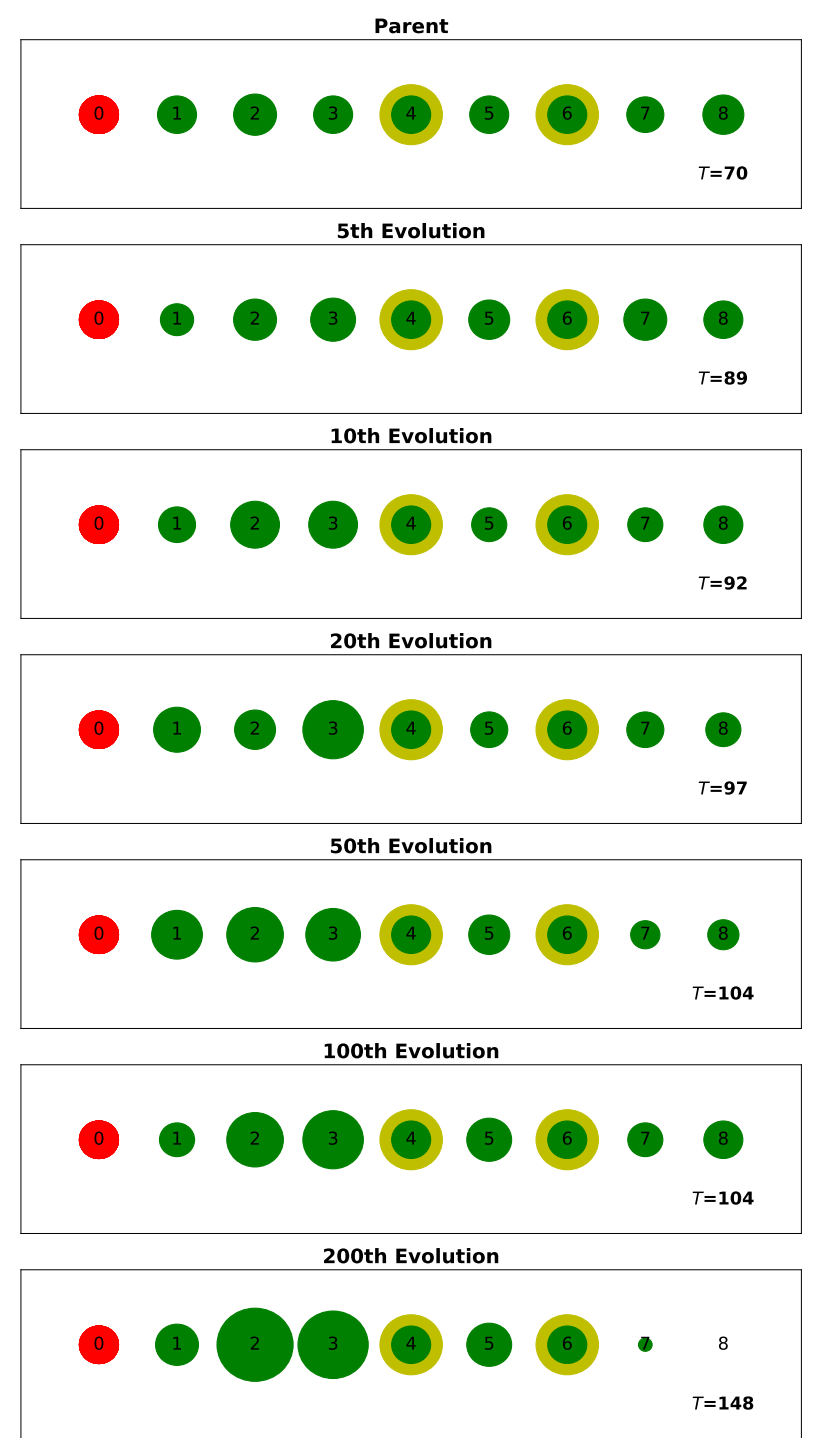

Fig. 6 - Snapshots of the resource distribution and total transmission, $T$ after different evolution counts. the total number of dropped messages or maximize the total number of transmissions. Note that there might be other communication-related goals such as faster communication or communicating with fewer resources, however, for the sake of simplicity, we only focus on the maximum number of dropped messages from sensor nodes to the sink for this problem. As a result, organisms with a lower number of drops are selected to create offspring. Since we consider the organisms individually, we assume they do not interact with each other.

Without loss of generality, we assume that sensor nodes have unlimited resources, i.e., they safely outlast the relay nodes. Without this assumption, dried-up sensor nodes might stop creating messages before relay nodes stop transmitting them. Hence, such an assumption helps us only to focus on relaying the messages instead of their creation. This assumption is also justified because sensor nodes with extra hardware must be larger than the relay nodes.

Our final assumption is that each node knows the location of the sink and other nodes to a reasonable degree. Thus, nodes do not relay messages coming from a node closer to the sink than they are, as described in Algorithm 1. This assumption is justified as well since each node can include a stamp on the messages allowing the receiving nodes to learn the last transmitting node.

\subsection{Analytic solution}

Although most resource management problems do not have an easy analytic solution, we can attempt to find one for this problem because of its simple geometry. To this end, we evaluate the resource distribution of an optimal organism, using Shapley Values of individual nodes for the organism. A Shapley Value is defined as the performance difference in a system with and without the part under in- vestigation. In other words, we can find the Shapley Value of node $n_{i} \in \mathcal{R}$ using the formula

$$
V\left(n_{i}\right)=P\left(\forall n_{j} \in \mathcal{R}\right)-P\left(\forall n_{j} \in \mathcal{R}-n_{i}\right),
$$

where $V\left(n_{i}\right)$ is the Shapley Value of node $n_{i}$ and $P(\mathcal{X})$ is the cumulative performance of all members of set $\mathcal{X}$.

Firstly, since they are located to the opposite side of the sink, i.e., upstream of all sensor nodes, $n_{7}$ and $n_{8}$ do not relay any messages. Hence,

$$
V\left(n_{7}\right)=V\left(n_{8}\right)=0 .
$$

For the remaining relay nodes, we employ the probability of a drop to measure their performance.

$$
V\left(n_{i}\right)=p_{\text {drop }}\left(\mathcal{R}-n_{i}\right)-p_{\text {drop }}(\mathcal{R}),
$$

where $p_{\text {drop }}(\mathcal{X})$ is the drop probability within the set $\mathcal{X}$. Note that since (5) focuses on drop performance, it is multiplied by a factor of -1 , i.e., performance is measured as the negative of the drops. 
Without loss of generality, we assume that transition between adjacent nodes is a certainty while there is still a non-zero chance of the next node receiving the message, as depicted in Fig. 2. The simulation parameters are also chosen to satisfy this assumption. Therefore, we expect no drop to occur if all nodes are present, which allows us to find $p_{\text {drop }}(\mathcal{R})=0$. Hence, only the first term of (5) remains.

We know that drops occur only if no other node receives the message. Otherwise, one of the receiving nodes, namely, $n_{i} \in \mathcal{R S}$ relays the message to the sink. Hence,

$$
p_{\text {drop }}(i)=\lambda_{i+1}\left(1-\prod_{k=2}^{i+1}\left(1-p_{k}\right)\right)
$$

where $\lambda_{i}$ is the activation rate of $n_{i}$ and $p_{k}$ is the probability of a successful transmission between node $n_{i}$ and $n_{i+k}$. Note that, $1-\prod\left(1-p_{k}\right)$ term gives the probability that no downstream node including the sink receives the message coming from $n_{i+1}$ and if $\lambda_{i+1}=0$ if $n_{i+1} \notin \mathcal{S}+\mathcal{R}$. Here, we also used the fact that the chosen simulation parameters ensures transmission between adjacent nodes, which renders drops possible only when one-step upstream node, i.e., $n_{i+1}$ if $p_{d r o p}(i)$ is investigated, is transmitting.

Activation rates depend on the position of the node. While sensor node activation occurs independent of other nodes, relay nodes depend on the activation of sensor nodes. For example, $n_{5}$, which only has the burden of transmitting messages from $n_{6}$, is activated less than $n_{1}$ $n_{3}$. As a result, $\lambda_{i}$ s can be solved recursively.

For a given node, we can find $\lambda_{i+1}$ as

$$
\begin{aligned}
\lambda_{i+1}= & \sum_{j>i+1, j \in \mathcal{S}+\mathcal{R}} \lambda_{j}\left(\sum_{\substack{n_{0}, n_{i} \notin \mathcal{R S} \\
n_{i+1} \in \mathcal{R S}}} \frac{1}{\mathcal{C}(\mathcal{R S})} p_{\mathcal{R S}}\right) \\
& +\sum_{n_{j} \in \mathcal{S}} \delta_{i+1, j} \lambda_{i+1}^{S},
\end{aligned}
$$

where $\delta_{i j}$ is the Kronecker Delta, $\lambda_{i}^{S}$ is the sensor activation rate of sensor node $n_{i}, n_{0}$ is the sink and $p_{R S}$ is the probability of obtaining the particular $\mathcal{R} \mathcal{S}$. It is calculated by

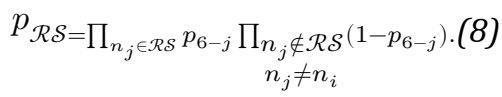

Although seemingly complicated, the first term of (7) describes the node acting as a relay probability by summing over all nodes, which might be relaying a message to node $n_{i}$. The second term describes the node acting as a sensor node. Note that second term becomes non-zero only when $n_{j} \in \mathcal{S}$.
Table 1 - Percent Shapley Values and resource distribution

\begin{tabular}{c|cc|c|c}
\multicolumn{3}{c|}{ Analyic Results } & \multicolumn{2}{c}{ Experimental Results } \\
\hline Node & Percent & Value & Percent & Value \\
\hline $\mathbf{1}$ & 29.72 & 35.67 & 21.09 & 27 \\
$\mathbf{2}$ & 27.80 & 33.36 & 28.91 & 37 \\
$\mathbf{3}$ & 28.18 & 33.82 & 30.47 & 39 \\
$\mathbf{5}$ & 14.30 & 17.16 & 14.84 & 19 \\
$\mathbf{7}$ & 0 & 0 & 4.69 & 6 \\
$\mathbf{8}$ & 0 & 0 & 0 & 0
\end{tabular}

\subsection{Evolutionary solution}

In the previous subsection, we used the simplicity of geometry to find an analytic solution to an otherwise complicated problem. In this section, we allow the parent organism to continuously evolve as described in Section 2.3.

We can see in Fig. 6 that expectedly, the resources allocated to $n_{7}$ and $n_{8}$ are reallocated to the $n_{2}$ and $n_{3}$, even after a few iterations. $n_{5}$, which only has the burden of relaying messages originated at $n_{6}$, possesses fewer resources than the $n_{2}$ and $n_{3}$, relaying messages originated at both of the sensor nodes.

Inspecting Fig. 5, we observe that evolution does not guarantee increased performance after each iteration. This observation coincides with the fact that the evolution of species does not guarantee better offspring at every generation. Furthermore, similar to its counterpart in biology, evolution may be influenced by successful organisms with suboptimal resource distribution. For example, since the sensors are activated randomly, an organism with higher $n_{4}$ sensor activity would require fewer resources overall than an organism with higher $n_{6}$ sensor activity.

Note that there is also a systematic error in our simulation method promoting decreased performance in some iterations. We force an infinitesimal change in all nodes in the organism, i.e., nodes do not die off when they do not have any resources in an iteration. As a result, nodes having no reservoir either stay at no resources or slightly go up, causing the organism to approach a distribution just below the optimal.

Table 1 shows the comparison between analytical and evolutionary results after 200 iterations. Although they all start at resources enough for 20 transmissions, as expected, $n_{7}$ and $n_{8}$ lose their resources while other nodes thrive during the evolutionary process. However, as explained above, even though it approaches zero, $n_{7}$ is not yet quite zero, wasting some resources, which should be reallocated to other nodes. The only dramatic discrepancy is $n_{1}$ being lower than the expectations by the analytical solution. We expect that the discrepancy diminishes with an increasing iteration count. 


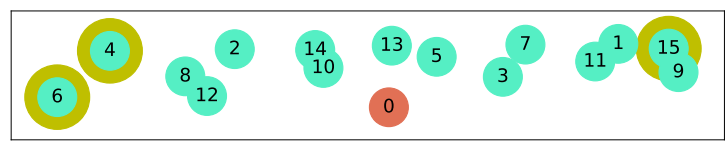

Fig. 7 - Layout for the simulation organism.

Table 2 - Simulation parameters

\begin{tabular}{l|l|l}
\multicolumn{1}{c}{$\begin{array}{c}\text { Name } \\
\text { Vesicle size }\end{array}$} & 40000 & Ualue \\
\hline Diffusion constant & $1 \times 10^{-12}$ & $\mathrm{~m}^{2} / \mathrm{s}$ \\
\hline Radius & 0.5 & $\mu \mathrm{m}$ \\
\hline Exhausted node ratio & 0.2 & \\
\hline \# of Vesicles per node & 40 & \\
\hline Offsprings & 2 & \\
\hline Node count & 16 & \\
\hline Dimensions & $1 \times 2 \times 10$ & $\mu m \times \mu m \times \mu m$ \\
\hline Change amplitude & 3 & \\
\hline
\end{tabular}

\section{VESICLE COUNT OPTIMIZATION}

In this section, we use our approach to optimize a randomly generated organism. The only design constraint we have is introducing a separation between the sink and the sensors, i.e., the probability of any sensor successfully sending information to the sink without the relay nodes is small enough to be ignored. The simulation parameters and the simulation organism are given in Table 2 and Fig. 7 respectively. The selected simulation parameters are all arbitrary; however, one can alter the time scale, diffusion constant, radii, and the organism size easily to fit them to an actual organism.

Firstly, we investigate the effect of the number of offspring per organism. Since the population is kept constant, the selection rate is proportional to the inverse of the offspring per organism.

\section{Child count change}

Changing the child count for each stage has dramatic effects on the performance of the organism. These effects are visible in Fig. 8. A smaller number of offspring imply that more parents join in the creation of the next generation. As a result, there is less uncertainty in the next generation. More offspring increase the uncertainty. If one of the parents reached their performance mostly due to luck, most of the offspring in the next generation becomes inferior. This situation leads to huge discrepancies in performance between generations.

\section{Amplitude change}

Increasing the amplitude of change inter-generations has a dramatic impact on the system performance. For large amplitudes, the evolution fails to reach its potential. Once the organism reaches a certain performance, the huge

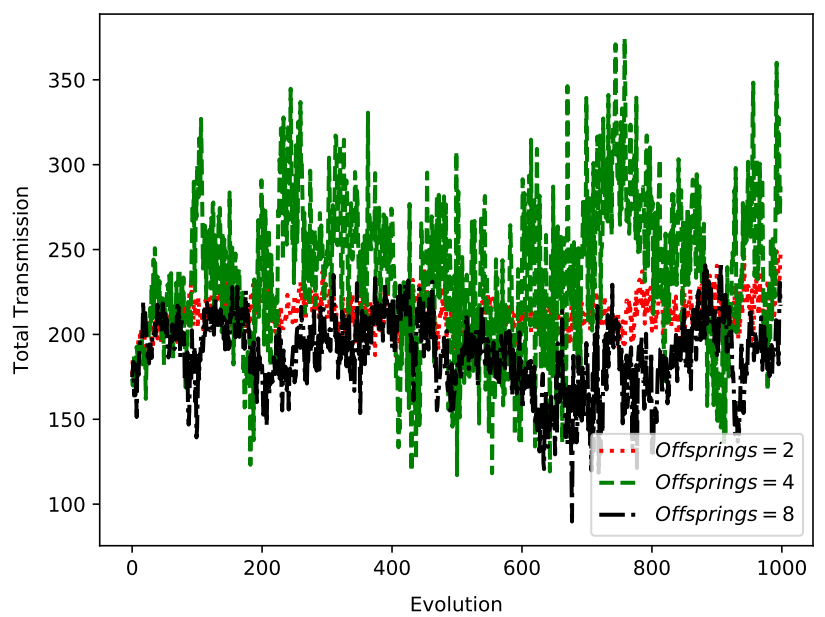

Fig. 8 - Performance of evolution with different child numbers.

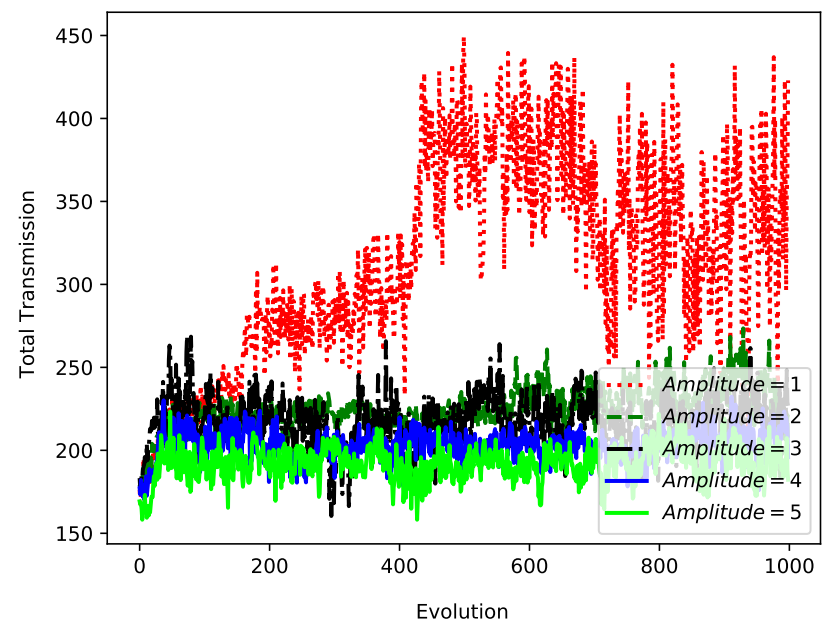

Fig. 9 - Performance of evolution with different amplitude values.

changes in the resource distribution set the organism back. Fig. 9 displays the performance of evolution with different change amplitudes.

Inspecting Fig. 9, we also realise that the performance of Amplitude $=1$ line is inferior for the first hundred iterations of evolution. This is obviously due to the small increases due to the limited inter-generational changes. However, the same factor boosts the final performance of the organism, which verifies the biological evolution, i.e., small changes advance the organisms while huge changes are not sustainable.

\section{CONCLUSION}

In this paper, we simulate the resource allocation in an organism, having nodes communicating via MC, using evolutionary game theory. We propose a two-staged evolution process realized by selecting the organisms with 
better transmission count and the creation of their offspring. Furthermore, we apply this approach to a simplistic MCN scenario, for which we also obtain an analytical solution. We simulate the performance of evolution concerning different parameters. Our work offers a simple demonstration of nature's solution to the resource management problem.

We believe our approach applies to many problems other than resource allocation. It also holds the potential of optimizing several different parameters to a reasonable degree. As future work, we will employ our method for node orientation, vesicle size, and receptor locations on the node surfaces.

\section{ACKNOWLEDGEMENT}

This work was supported in part by the AXA Research Fund (AXA Chair for Internet of Everything at Koç University) and Huawei Graduate Research Scholarship.

\section{REFERENCES}

[1] Ian F Akyildiz, Max Pierobon, Sasi Balasubramaniam, and Y Koucheryavy. "The internet of bionano things". In: IEEE Communications Magazine 53.3 (2015), pp. 32-40.

[2] Ozgur Baris Akan, Hamideh Ramezani, Meltem Civas, Oktay Cetinkaya, Bilgesu Arif Bilgin, and Naveed Ahmed Abbasi. "Information and communication theoretical understanding and treatment of spinal cord injuries: State-of-the-art and research challenges". In: IEEE Reviews in Biomedical Engineering (2021).

[3] Ozgur B Akan, Hamideh Ramezani, Tooba Khan, Naveed A Abbasi, and Murat Kuscu. "Fundamentals of molecular information and communication science". In: Proceedings of the IEEE 105.2 (2016), pp. 306-318.

[4] Caglar Koca, Meltem Civas, Selin M. Sahin, Onder Ergonul, and Ozgur B. Akan. "Molecular Communication Theoretical Modeling and Analysis of SARSCoV2 Transmission in Human Respiratory System". In: IEEE Transactions on Molecular, Biological and Multi-Scale Communications (2021), pp. 1-1. DoI: 10.1109/TMBMC . 2021.3071748.

[5] Saswati Pal, Nabiul Islam, Sudip Misra, and Sasitharan Balasubramaniam. "In Vivo Channel Characterization for Dengue Virus Infection". In: Proceedings of the Sixth Annual ACM International Conference on Nanoscale Computing and Communication. 2019, pp. 1-7.

[6] Meltem Civas and Ozgur B Akan. "Rate of Information Flow Across Layered Neuro-Spike Network in the Spinal Cord". In: IEEE transactions on nanobioscience 19.3 (2020), pp. 368-377.
[7] Murat Kuscu, Ergin Dinc, Bilgesu A Bilgin, Hamideh Ramezani, and Ozgur B Akan. "Transmitter and receiver architectures for molecular communications: A survey on physical design with modulation, coding, and detection techniques". In: Proceedings of the IEEE 107.7 (2019), pp. 1302-1341.

[8] Murat Kuscu and Ozgur B Akan. "Detection in Molecular Communications with Ligand Receptors under Molecular Interference". In: arXiv preprint arXiv:2101.00081 (2020).

[9] Murat Kuscu and Ozgur B Akan. "Channel sensing in molecular communications with single type of ligand receptors". In: IEEE Transactions on Communications 67.10 (2019), pp. 6868-6884.

[10] Vahid Jamali, Arman Ahmadzadeh, Wayan Wicke, Adam Noel, and Robert Schober. "Channel modeling for diffusive molecular communication A tutorial review". In: Proceedings of the IEEE 107.7 (2019), pp. 1256-1301.

[11] Weisi Guo, Taufiq Asyhari, Nariman Farsad, H Birkan Yilmaz, Bin Li, Andrew Eckford, and ChanByoung Chae. "Molecular communications: Channel model and physical layer techniques". In: IEEE Wireless Communications 23.4 (2016), pp. 120127.

[12] Arman Ahmadzadeh, Adam Noel, and Robert Schober. "Analysis and design of multi-hop diffusion-based molecular communication networks". In: IEEE Transactions on Molecular, Biological and Multi-Scale Communications 1.2 (2015), pp. 144-157.

[13] Satish K Tiwari, Tadi Ravi Teja Reddy, Prabhat K Upadhyay, and Daniel Benevides Da Costa. "Joint optimization of molecular resource allocation and relay positioning in diffusive nanonetworks". In: IEEE Access 6 (2018), pp. 67681-67687.

[14] Yuting Fang, Adam Noel, Nan Yang, Andrew W Eckford, and Rodney A Kennedy. "Symbol-by-symbol maximum likelihood detection for cooperative molecular communication". In: IEEE Transactions on Communications 67.7 (2019), pp. 4885-4899.

[15] Xuan Chen, Miaowen Wen, Chan-Byoung Chae, LieLiang Yang, Fei Ji, and Kostromitin Konstantin Igorevich. "Resource Allocation for Multi-User Molecular Communication Systems Oriented to The Internet of Medical Things". In: IEEE Internet of Things Journal (2021).

[16] Chunxiao Jiang, Yan Chen, and KJ Ray Liu. "Nanoscale molecular communication networks: a game-theoretic perspective". In: EURASIP Journal on Advances in Signal Processing 2015.1 (2015), pp. 1-15. 
[17] Caglar Koca and Ozgur B Akan. "Anarchy versus cooperation on internet of molecular things". In: IEEE Internet of Things Journal 4.5 (2017), pp. 14451453.

[18] Adam Noel, Yuting Fang, Nan Yang, Dimitrios Makrakis, and Andrew W Eckford. "Effect of local population uncertainty on cooperation in bacteria". In: 2017 IEEE Information Theory Workshop (ITW). IEEE. 2017, pp. 334-338.

[19] Apostolos Almpanis, Christophe Corre, and Adam Noel. "Agent Based Modeling of the Rhizobiome with Molecular Communication and Game Theory". In: Proceedings of the Sixth Annual ACM International Conference on Nanoscale Computing and Communication. 2019, pp. 1-7.

[20] John Maynard Smith. Evolution and the Theory of Games. Cambridge university press, 1982.

\section{AUTHORS}

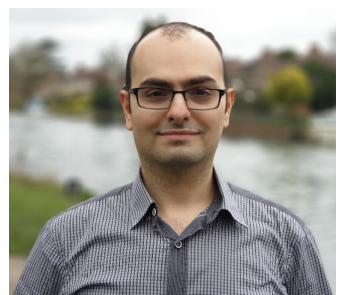

Caglar Koca received a B.Sc. degree in electrical and electronics engineering from Middle East Technical University, Ankara, Turkey, in 2010, an M.Sc. degree in electrical and electronics engineering from Koç University, Istanbul, Turkey, in 2014, and is currently pursuing a Ph.D. degree at University of Cambridge. He joined the Next-Generation and Wireless Communication Laboratory (NWCL), Koç University in 2011 and he is currently a research assistant at the Internet of Everything (IoE) group, University of Cambridge since 2017. His research interests include Internet of Everything, information theory and molecular communications.

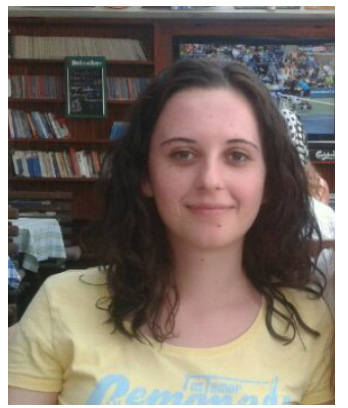

Meltem Civas received her B.S. degree in Electrical and Electronics Engineering in Bilkent University, Ankara, Turkey, in January 2016, and her M.S. degree at the Electrical and Electronics Engineering Department, Koç University, Istanbul, Turkey in September 2018. She is currently a research assistant at the Next-generation and Wireless Communications Laboratory and pursuing her Ph.D. degree in Electrical and Electronics Engineering, Koç University. Her research interests include Internet of Everything and molecular communications.

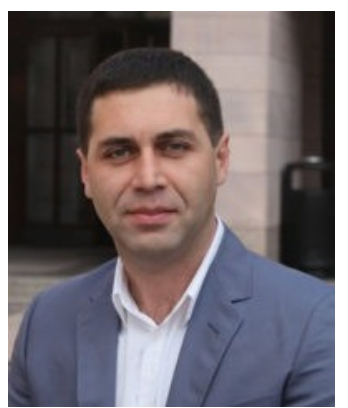

Ozgur B. Akan received his Ph.D. degree in electrical and computer engineering from the Broadband and Wireless Networking Laboratory, School of Electrical and Computer Engineering, Georgia Institute of Technology, Atlanta in 2004. He is currently with the Electrical Engineering Division, Department of Engineering, University of Cambridge, United Kingdom, and also the director of the Next-Generation and Wireless Communications Laboratory in the Department of Electrical and Electronics Engineering, Koç University. His research interests include wireless, nano, and molecular communications, and the Internet of Everything. 